\title{
A New Era in Acute Ischaemic Stroke Treatment: A Review of UK and European Thrombectomy Guidelines
}

\author{
Syeda Nadia Nadeem ${ }^{1}$ and Alakendu Sekhar ${ }^{2}$ \\ ${ }^{1}$ University of Liverpool Medical School, UK \\ ${ }^{2}$ The Walton Centre NHS Foundation Trust, UK \\ Corresponding author: Syeda Nadia Nadeem, University of Liverpool Medical School, UK, Tel: +44 (0) 7940323860; E-mail: \\ snadnadeem@googlemail.com
}

Received: Jun 16, 2016; Accepted: Oct 26, 2016; Published: Oct 31, 2016

Citation: Nadeem SN, Sekhar A. A New Era in Acute Ischaemic Stroke Treatment: A Review of UK and European Thrombectomy Guidelines. J Neurol Neurosci. 2016, 7:5.

\section{Abstract}

Background: Acute ischemic stroke is the second leading cause of death worldwide. It creates a massive burden not only on the patient's life but society as a whole. There has been much advancement in stroke management over the past few decades but 2015 has shown giant leaps in this. Up till now, thrombolysis was the main stroke treatment but recently several randomized controlled trials have emerged to demonstrate the efficacy behind using thrombectomy and how it will lead us into the future by treating ischemic stroke quicker and more effectively than ever before.

Aim: To perform a systematic review of the new trials that compare thrombectomy to best medical-care. Furthermore, to critically analyze the current thrombectomy guidelines across UK and Europe in order to develop a thrombectomy best practice protocol that can be used as a guide worldwide.

Method: Numerous articles which highlight the use of thrombectomy compared to thrombolysis alone in acute ischemic stroke were reviewed. Most recent UK and Europe guidelines for thrombectomy use were compared and critically appraised.

Results and conclusion: Results show that thrombectomy used with thrombolysis reduces mortality rates and improves patients' functional independence and vessel reperfusion rates significantly from $37 \%$ to $100 \%$ compared to thrombolysis alone. Current European recommendations complies with these results and forms relevant guidance whereas, the UKs guidelines are incompatible, lacking structure and recent evidence. As a result, this review has created a new protocol which encompasses the trial results and European recommendations, in the hope to assist physicians worldwide.
Keywords: Acute ischaemic stroke; Thrombectomy; Thrombo-embolisms

\section{Abbreviations}

ASPECTS score: Alberta Stroke Program Early CT Score; ATP: Adenosine Triphosphate; BMI: Body Mass Index, measure in $\mathrm{kg} / \mathrm{m}^{2}$; CBF: Cerebral Blood Flow; CPP: Cerebral Perfusion Pressure; CT: Computed Tomography; CTA: Computed Tomography Angiography; CVR: Cerebral Vascular Resistance; $\mathrm{DH}$ : Decompressive Hemicraniotomy; ESO: European Stroke Association; EXTEND-IA: Extending the Time for Thrombolysis in Emergency Neurological Deficits with Intra-Arterial Therapy; GCA: Giant Cell Arteristis; IVT: Intravenous Thrombolysis; MCR: Mechanical Clot Retraction/Retrieval; MR CLEAN: Multicentre Randomized Clinical Trial of Endovascular Treatment in the Nethelands, ESCAPE; MRI: Magnetic Resonance Imaging; mRS: Modified Rankin Score; NICE: The National Institute for Health and Care Excellence; NIHSS: National Institute of Health Stroke Scale; NNT: Number Needed to Treat; PAN: Polyarteritis Nodosa; PISTE: Pragmatic Ischaemic Stroke Thrombectomy Evaluation; RA: Rheumatoid Arthritis; RCP: Royal College of Physicians; RCT: Randomized Controlled Trial; SLE: Systemic Lupus Erythematosus; SWIFT PRIME: Solitaire with the Intention for Thrombectomy as Primary treatment for Acute Ischemic Stroke; TIA: Transient Ischaemic Attack

\section{Introduction}

\section{Acute ischaemic stroke}

Stroke definition and statistics: An updated definition of ischaemic stroke by the American Stroke Association is 'an episode of neurological dysfunction caused by focal cerebral, spinal, or retinal infarction' where infarction is 'cell death due to ischaemia' and is evidenced either pathologically or by clinical symptoms lasting longer than 24 hours [1]. 
Almost 152,000 strokes occur per year in the UK, of which the majority $(85 \%)$ is of ischaemic origin [2]. This means that every three and a half minutes, someone in the UK suffers a stroke, and almost half of these people are left with a life changing disability [3].

It is the fourth biggest cause of death in the UK and the second leading cause worldwide [2]. As a result, there is a massive economic burden of stroke on society and the NHS. It is estimated that stroke costs our society $f 9$ billion a year; this includes stroke treatment and rehabilitation as well as the loss in workforce and productivity [4]. Stroke treatment consumes $5 \%$ of the entire NHS treatment costs and an astonishing $50 \%$ of the total direct care costs (i.e. diagnosis, inpatient and outpatient care) [4].

\section{Pathophysiology of ischaemic stroke}

Our brain has a very high metabolic rate; it consumes $20 \%$ of our total oxygen supply and requires $15 \%$ of our cardiac output [5]. Neurons do not store energy in the form of glycogen like other tissue cells therefore they rely completely on a constant supply of glucose and oxygen provided by cerebral blood flow (CBF) for its metabolic demands [6]. Anything which grossly disrupts this supply can cause hypoxia, ischaemia and if severe or prolonged, can result in irreversible infarction.

Normal CBF to grey matter is approximately $100 \mathrm{ml} / 100 \mathrm{~g} /$ min; this is well controlled by cerebral perfusion pressure (CPP) and cerebral vascular resistance (CVR) [7]. If CBF falls to $0 \mathrm{ml} / 100 \mathrm{mg} / \mathrm{min}$, irreversible cerebral infarction occurs in 4-10 mins, this is prolonged to 1 hour is CBF is between $16-18$ $\mathrm{ml} / 100 \mathrm{mg} / \mathrm{min}$ [7]. However, if CBF is maintained above 20 $\mathrm{ml} / 100 \mathrm{mg} / \mathrm{min}$, reversible ischaemia without infarction takes place [7]. Factors that disrupt CPP or CVR can result in an ischaemic stroke.

Generally there are two types of ischaemia; global and focal [5]. Global ischaemia results from a drop in CPP due to a decline in systolic blood pressure to below $50 \mathrm{mmHg}$, such as in severe hypotension, shock or cardiac arrest; this results in extensive brain injury [5]. However, in strokes cases, we typically see focal ischaemia, due to an increase in CVR from atherosclerosis and thromboembolisms [5].

Once cerebral blood flow is occluded, many processes take place leading to ischaemia. A lack of glucose and oxygen stops production of ATP that is needed for active transport across ion channels, therefore, an intracellular influx of sodium and calcium ions occurs $[7,8]$. This triggers several reactions including intracellular proteolysis, excessive release of glutamate across synapses resulting in neurotoxicity, and production of prostaglandins and free radicals, all of which lead to neuronal damage and necrosis $[7,8]$.

\section{Aetiology of ischaemic stroke}

The main causes of focal ischaemic stroke relate to 'Virchows triad' of thrombus formation, which states that a thromboembolism occurs when any one of these three factors exist; endothelial damage (e.g. by hypertension), abnormal blood flow (including blood stasis or turbulent flow, e.g. by atheromatous plaque), and blood hypercoaguablilty [5]. We can classify these pathological factors into the following causes as summarised in the table below (Table 1).

Table 1 Causes of thromboembolisms.

\begin{tabular}{|l|l|l|}
\hline \multicolumn{2}{|l|}{ Causes of Thromboembolisms } \\
\hline Thrombosis & Embolism & Other \\
\hline $\begin{array}{l}\text { Atheromatous plaque (Risk } \\
\text { factors include: }\end{array}$ & Cardiac: & - Hypercoaguable blood (e.g. Protein S or C deficiency, Sickle Cell Disease, dehydration etc.) \\
- Obesity (>30 BMI) & - Myocardial infarction & - Vasculitis (e.g. PAN, Wegener's granulomatosis, GCA etc) \\
- Diabetes & - Valvular disease & - Collagen disorders and inflammatory conditions (e.g. RA, SLE) \\
- Smoking & - Atrial fibrillation & \\
- Hyperlipidaemia & - Endocarditis & \\
- Hypertension) & Venous: & \\
& - Deep Vein Thrombosis & \\
& Fat embolism: & \\
& - Bone Trauma & \\
\hline
\end{tabular}

\section{Classifications and clinical features}

Ischaemic stroke can be classified clinically using the Bamford/Oxford Stroke Classification (Table 2) [9-11]. This splits stroke up into syndromes based on clinical signs and symptoms from which we can predict the location of the insult and vessel territory as well as the extent and prognosis of the ischaemia [9].

Table 2 Bamford/Oxford stroke classification.

Bamford/Oxford Stroke Classification

Stoke syndromes

Stoke syndromes

TACS

PACS

POCS

LACS 


\begin{tabular}{|c|c|c|c|c|}
\hline & $\begin{array}{l}\text { (Total Anterior Circulation } \\
\text { Stroke) } \\
\text { Involves ACA and MCA }\end{array}$ & $\begin{array}{l}\text { (Partial Anterior Circulation } \\
\text { Stroke) } \\
\text { Involves only ACA or MCA }\end{array}$ & $\begin{array}{l}\text { (Posterior Circulation } \\
\text { Stroke) } \\
\text { Involves PCA/Basilar/ } \\
\text { Vertebral arteries }\end{array}$ & $\begin{array}{l}\text { (Lacunar Circulation Stroke) } \\
\text { Involves small vessels in the } \\
\text { subcortical area }\end{array}$ \\
\hline Clinical Features & $\begin{array}{l}\text { Features must include ALL } \\
\text { of: } \\
\text { - Motor and Sensory } \\
\text { defect } \\
\text { - Homonymous } \\
\text { hemianopia } \\
\text { - Higher cerebral } \\
\text { dysfunction (dysphasia, } \\
\text { visuospatial deficit, } \\
\text { reduced a } \\
\text { consciousness) }\end{array}$ & 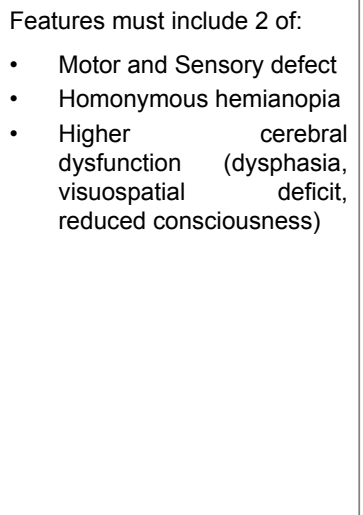 & $\begin{array}{l}\text { Features must include } \\
\text { any of: } \\
\text { - Brainstem or } \\
\text { cerebellar } \\
\text { dysfunction } \\
\text { - Isolated } \\
\text { homonymous } \\
\text { hemianopia } \\
\text { - Impaired } \\
\text { consciousness } \\
\text { Bilateral sensory/ } \\
\text { motor dysfunction } \\
\text { Ipsilateral cranial } \\
\text { nerve palsy and } \\
\text { contralateral } \\
\text { weakness. }\end{array}$ & $\begin{array}{l}\text { Features must include any } \\
\text { of: } \\
\text { - Only motor defects } \\
\text { - Only sensory defects } \\
\text { - Mixed motor \& sensory } \\
\text { defects } \\
\text { - Ataxic hemiparesis } \\
\text { (hemiparesis cerebellar } \\
\text { ipsilateral car } \\
\text { ataxia) }\end{array}$ \\
\hline Prog. & \multicolumn{3}{|c|}{ Prognosis: Mortality rate at $\mathbf{3 0}$ days: } & \\
\hline & $39 \%$ & $4 \%$ & $7 \%$ & $2 \%$ \\
\hline
\end{tabular}

Ischaemic stroke is clinically diagnosed using the clinical features mentioned above. Imaging techniques e.g. CT or MRI scans are carried out to confirm this diagnosis and exclude haemorrhage and other causes. Further tools are used to assess extent of stroke, such as the NIHSS (National Institute of Health Stroke Scale) and mRS (Modified Rankin Score). The

\section{Current stroke management}

The current management pathway set out by NICE and the Royal college of Physicians for acute stroke is summarized in the Figure $1[14,15]$. NIHSS is done at baseline and throughout hospital stay to determine the severity of stroke and prognosis [12]. The mRS determines the effect of the stroke on a patient's functioning, i.e. the degree of disability [13].

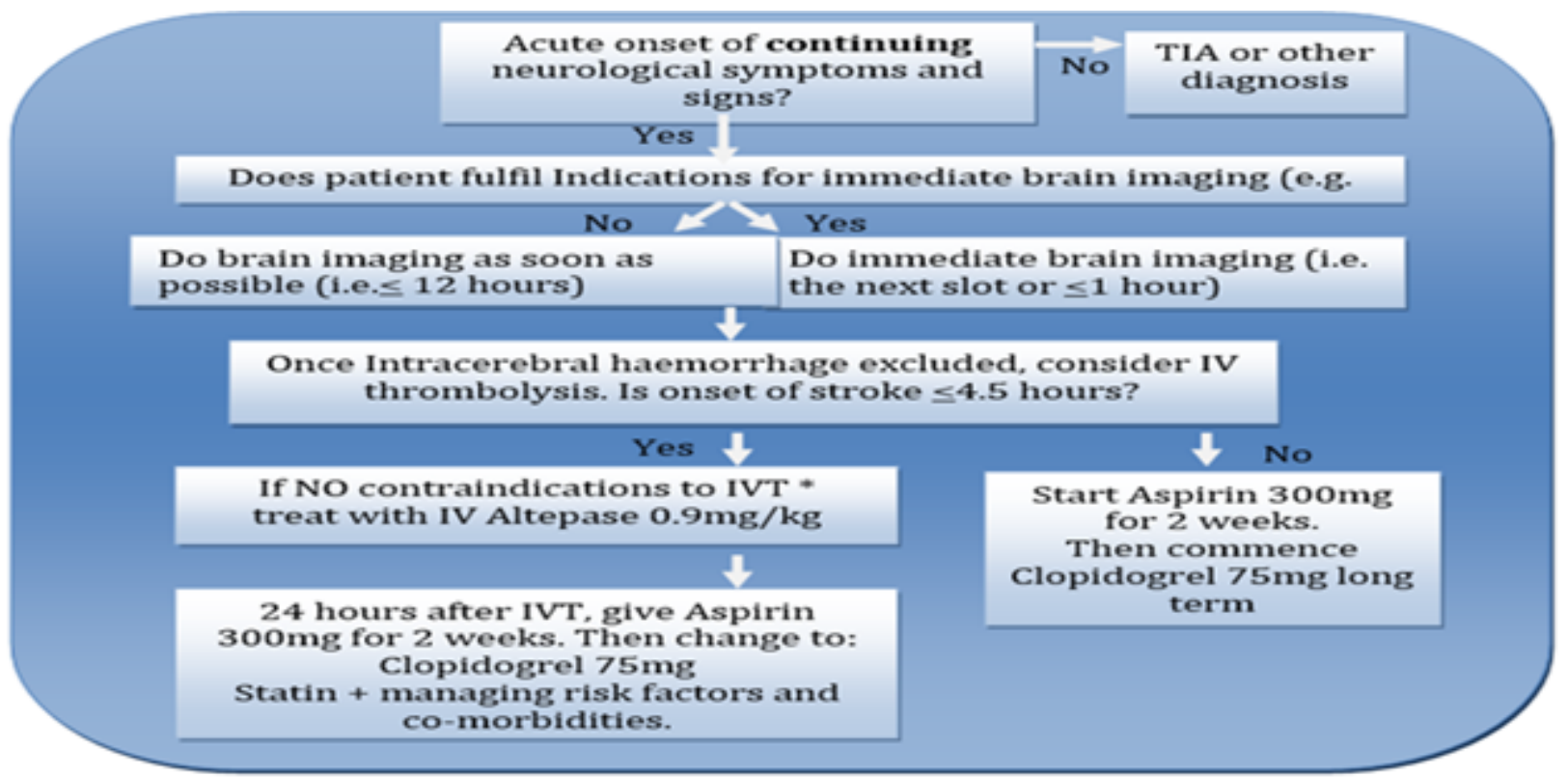

Figure 1 The current management pathway set out by NICE and the Royal college of Physicians for acute stroke.

*Indications for 'immediate' brain imaging [14]:

- Indications for thrombolysis or early anticoagulation treatment.
- On anticoagulant treatment.

- A known bleeding tendency. 
- A depressed level of consciousness (Glasgow Coma Score below 13).

- Unexplained progressive or fluctuating symptoms.

- Papilloedema, neck stiffness or fever.

- Severe headache at onset of stroke symptoms.

Invasive endovascular intervention, such as Thrombectomy, are also available, however, their use is not well promoted in the stroke guidelines and is only considered in the context of research or if IVT (intravenous thrombolysis) has failed or is unsuitable [15].

Due to the time delay and haemorrhagic complications of IVT use, thrombectomy was developed and approved in 2004 as an alternative or adjunctive treatment [16]. There are several devices for endovascular intervention including coil retrievers, vacuum aspiration (Penumbra system) and stent retrievers. Stent retrievers were approved in 2012 and are the most widely used device, with the newest generation of 'Solitaire' Stent Retrievers preferred due to lower risk of complications and greater efficacy [17].

The actual procedure involves inserting a guidewire, balloon catheter and stent retriever through the femoral artery to the occluded vessel. Once the stent retriever is deployed under angiographic imaging, the thrombus is trapped, aspirated and removed [18]. Thrombectomy works best for large or proximal vessel occlusions [17]. The aim puncture-to-perfusion time of < $1 \mathrm{~h}$ hour and should be done within 90 mins after IVT [19]. Complications of thrombectomy include, symptomatic intracranial haemorrhage ( $\mathrm{SICH})(2-4 \%)$, distal emboli (2\%), arterial dissection (1\%), vessel perforation (1\%) and access site complications (e.g. groin infection or haemorrhage (1\%)) $[17,20]$. These risks are much lower than those for IVT alone and become even lower, as the device technology develops.
In summary, thrombectomy is a rapidly developing field within stroke management. There are numerous trials researching its efficacy compared to IVT alone or as an adjunct. The interest in thrombectomy is rising with the opinion that it could soon replace IVT altogether, therefore, it is imperative that this should be matched by a clear and comprehensive guidance in the UK [21].

\section{Aim of the Review}

To compare and critically analyse the current UK and European guidelines on thrombectomy use in order to create a new and updated guideline proposal.

\section{Objectives}

1. To compare UK and European guidance on thrombectomy.

2. To analyze and discuss the evidence-based and limitations of the current recommendations.

3. To suggest proposal for best practice.

\section{Discussion}

Currently, there are two main evidence based recommendations for mechanical clot retrieval: UK NICE guidance, 'Mechanical clot retrieval for treating acute ischaemic stroke', July 2013, and a 'Consensus statement on mechanical thrombectomy in acute ischaemic stroke' by the ESO (European Stroke Association), which was last updated in February $2015[19,20]$.

Table 3 Selection criteria for thrombectomy.

\begin{tabular}{|c|c|c|}
\hline & NICE & ESO \\
\hline Indications & $\begin{array}{l}\text { - Patients in whom thrombolysis is unsuitable. } \\
\text { - Patients in whom thrombolysis has failed. } \\
\text { thrombectomy can only be carried out for research } \\
\text { purposes in the form of clinical trials. }\end{array}$ & $\begin{array}{l}\text { Patients with acute ischaemic stroke who have had IVT } \\
\text { within } 4.5 \text { hours, with large artery occlusion, in the } \\
\text { anterior circulation or basilar artery, presenting }<6 \text { hours } \\
\text { after symptom onset. } \\
\text { IVT unsuitable or contraindicated (e.g. raised INR due to } \\
\text { Warfarin treatment) } \\
\text { - For the purpose of clinical trials }\end{array}$ \\
\hline Contra-indications & - Patients in whom thrombolysis is suitable & $\begin{array}{l}\text { - Radiological sign of large infarcts (e.g. ASPECTS score } \\
0-4 \text { ) }\end{array}$ \\
\hline
\end{tabular}

\section{Selection criteria for thrombectomy}

NICE's criteria is very vague and restricts the use of thrombectomy as a last resort therapy, probably due to its 'unproven efficacy'. It also fails to explain the definition of 'suitable' and 'unsuitable', and as a result, makes the guidance difficult to follow in clinical practice, especially in emergency stroke situations [22] (Table 3).

ESO guidance, on the other hand, has very clear outlines. It allows for a 6 hour time window from onset to reperfusion as evidenced by the MR CLEAN trial, which found that treatment was not effective after $6 \mathrm{hrs} 19$ mins [22]. Only patients with anterior circulation (including ICA, M1, M2 segment of MCA) and basilar occlusions are indicated because these patients are the only cases studied in the main trials [17,22-26]. Large vessel occlusion is indicated due to the fact that clot retrieval is only effective in large and proximal arteries due to the size of the device [17]. ESO makes it clear on how to identify large vessel occlusion, using CT-angiography, or if not available, an NIHSS $>9$ (within 3 hours from onset) or $>7$ (between 3-6 hours post-onset) $[19,27,28]$. An ASPECT score $0-4$ is contraindicated for the reason that evidence from MR CLEAN has shown that 
thrombectomy is only beneficial with ASPECT scores 5-10, not where there is extensive ischaemia $[19,22]$.

\section{Patient selection}

NICE does not explain how patients should be selected, only that it should be done by a stroke specialist clinician [20]. ESO recommends that patients should be selected on diagnosis of a large vessel occlusion using non-invasive imaging (e.g. CTangiography) or on a high NIHSS score ( $>9$ if within 3 hours from onset, or $>7$ between 3-6 hours post-onset), with an ASPECTS score $>519$. Patients should be selected by an MDT of a stroke physician and a neurointerventionalist [19]. ESO's detailed guidance ensures that the correct patients are selected by the most experienced team so that thrombectomy has the best chance for success with the lowest risk of harm.

\section{Procedure}

Both guidelines suggest that thrombectomy should be carried out using a 'stent retriever' by a neurointerventionalist and the patient should be under local or general anaesthaesia, depending on the patient.

\section{Conclusions on efficacy of thrombectomy}

NICE believe that the 'efficacy is unproven' for thrombectomy usage in acute ischaemic stroke, however, ESO supports the use of thrombectomy and states that there is 'very good evidence for early thrombectomy $[9,20]$.

This conclusion, by NICE that thrombectomy is ineffective, is based on results from studies that showed low thrombectomy recanalisation rates ( $41 \%)$, lack of significant improvement in functionality using $\mathrm{mRS}$ in thrombectomy compared to IVT alone, and similar mortality rates in those treated with thrombectomy alone versus thrombectomy as an adjunct to IVT (24\% and $18 \%$ respectively) [20,29-31].

However, ESO presents a very different picture using four very large multicentre trials including MR CLEAN, ESCAPE, SWIFT PRIME, EXTEND-IA, all of which find Thrombectomy (using stent retrievers) as an adjunct to be more effective than using IVT alone. MR CLEAN is the largest of the trials and aims to assess functional outcome using the mRS score, of thrombectomy + IVT therapy versus IVT alone. It concludes that there is a significant difference in the mRS at 90 days, with $\mathrm{mRS}$ of 3 in the intervention group (Thrombectomy + IVT) and 4 in the control (IVT only) group, with an odds ratio of 1.6722. This shows that if thrombectomy is used, patients suffer less disability and are able to walk without assistance [32]. The other three studies concur and add to these results:

- EXTEND-IA, a trial that looked at the reperfusion rates of intra-arterial clot retrieval after IVT compared to IVT alone, found reperfusion rate at 24 hours to be $100 \%$ (thrombectomy) compared to $37 \%$ (IVT alone), and a NNT of 3 to obtain mRS 0-2 at 90 days by thrombectomy (mRS of $0-2$ at 90 days: $71 \%$ intervention group, $40 \%$ control group)23.
- The ESCAPE trial compared rates of functional outcomes (mRS 0-2, NIHSS) and mortality in the intervention (thrombectomy + IVT) and control (IVT alone) groups. It found a large drop from baseline NIHSS of 16 to 6 at 24 hours in the intervention group. The control group did not improve as drastically (17 baseline to 13 at 24 hours) [24]. Both groups shifted from the category of 'moderate-severe stroke' to 'moderate stoke', however the thrombectomy group regained far more functionally, almost to a 'minor stroke' category [24,33].

- SWIFT PRIME, a trial with the aim of comparing long-term functional outcome at 90 days in intervention (thrombectomy + IVT) and control (IVT alone) groups. It found that although the mortality rate was not significantly lower $9 \%$ (intervention) versus $12 \%$ (control), the 90-day functional independence was much higher $160 \%$ intervention vs. $35 \%$ control) [34].

- In terms of safety, there is a reduction in mortality rates with thrombectomy across all four trials but only ESCAPE shows it to be statistically significant $10.4 \%$ (intervention) versus $19 \%$ (control) [24].

The primary outcome used for assessing efficacy is mRS score at 90 days. Table 4 provides an example of the disparity between results from evidence used by NICE and ESO to support their different views on efficacy and hence the guidance.

Table 4 An example of the disparity between results from evidence used by NICE and ESO to support their different views on efficacy and hence guidance.

\begin{tabular}{|l|l|l|l|}
\hline \multicolumn{3}{|c|}{ mRS of $\mathbf{0 - 2}$ (Functionally independent at $\mathbf{9 0}$ days) } \\
\hline $\begin{array}{l}\text { RCT used mRS 0-2 } \\
\text { at } 90 \text { days }\end{array}$ & $\begin{array}{l}\text { Thrombecto } \\
\text { my + IVT }\end{array}$ & $\begin{array}{l}\text { IVT } \\
\text { alone }\end{array}$ & Conclusion \\
\hline $\begin{array}{l}\text { IMS III } 2013 \text { trial } \\
\text { (used by NICE) 30 }\end{array}$ & $40.8 \%$ & $38.7 \%$ & $\begin{array}{l}\text { No significant } \\
\text { improvement- } 1.5 \% \\
\text { points }\end{array}$ \\
\hline $\begin{array}{l}\text { MR CLEAN 2015 } \\
\text { trial (used by ESO) } \\
22\end{array}$ & $32.6 \%$ & $19.1 \%$ & $\begin{array}{l}\text { Significant } \\
\text { improvement } \\
13.5 \% \text { points }\end{array}$ \\
\hline
\end{tabular}

The difference in these specific findings may be due to limitations of the IMS III trial, for example, the unequal assigning of patients into intervention and control group using a 2:1 ratio, lack of standardization in the use of endovascular device and technique as well as a higher baseline mRS in the intervention group. The MR CLEAN trial seems to be more reliable as it lacks these limitations and its results are supported by other robust trials.

As a whole, ESO recommendations seem much more robust as evidenced by the fact that its findings are from the most recent (2015) studies, all of which are randomised controlled trials, using the same stent retriever device with large sample sizes (e.g. 500 patients in MR CLEAN). In comparison, NICE uses a mixture of less reliable studies including meta-analysis, RCTs and case series, all of which are relatively old (ranging from 2011 to 2013) and use a range of different devices, all with different efficacy and safety rates [29-31]. 


\section{New guideline proposal for best practice}

The following suggested guidelines (Figure 2) have been created using current guidance from NICE and ESO, the evidence cited and the current thrombectomy protocol from the Walton Centre [19,20,35-40].

Once patient is elinically diagnosed with stoke carry out a:

- CT (exclude hacmorrhage) as soon as possible, using criteria for brain imaging*

- CT Angiography (to find large vessel ocelusion)

- Baseline NIHSS (to find large vessel ocelusion)

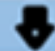

Administer IVT (Altepase $0.9 \mathrm{mg} / \mathrm{kg}$ ) $\leq 4.5 \mathrm{hrs}$ hospital

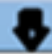

\section{Consider referral to specialist centre for thrombectomy using following criteria:} Indications

\section{Contraindications}

- IVT administered $\leq 4.5 \mathrm{hrs}$

- Large vessel occlusion (ICA, M1, M2 of MCA or Basil ax) diagnosed:

- on CTA or

- NIHSS $\geq 9$ (within 3 hours from onset) or $\geq 7$ (between $3-6$ hours post -onset).

- IVT contraindicated:

- raised INR (2-3) due to Warfarin

- TVT failed/refractory

- ASPECTS 5-10

- Able to attempt reperfusion $\leq 6$ hours

- In context of clinical trial
- Evidence of intracranial haem orrhage on CT

- Exclude ASPECTS $0-4$

- Vascular access contraindicated (e.g. fem oral bypass surgery)

- Allergy to contrast

Patient selection to be carried out by stroke physician and interventional neuroradiologist. Thrombectomy procedure:

- Carried out by interventional neuroradiologist, under supervison of stroke specialist neurologist

- Patient under local (LA) or general anaesthetic (GA). LA is preferred to GA as it has lower in-hospital mortality risks, but depends on the state of patient (e.g. if patients is agitated, GCS $<8$, breathing compromised, use $\mathrm{GA}^{36}$.

- Use stent retriever device

- Aim to complete procedure $\leq 1$ hour to reduce rate of complication ${ }^{37}$

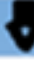

Immediately post-procedure: Monitor NIHSS, GCS and vital signs

24 hours post-procedure:

- Measure NIHSS

- Do CT and CTA (to exelude haemorrhagie complications and confirm vessel patency)

- Continue on the normal ischaemic stroke protocol from NICE:

- start patient on Aspirin $300 \mathrm{mg}$ (or Clopidogrel)

- Commence patient on long term antiplatelet therapy (Clopidogrel $75 \mathrm{mg}$ )

Discharge patient after consultant review and arrange for a follow up appointment in 3 months with stroke neurologist.

Figure 2 New guideline proposals for best practice.

\section{Limitations of new recommendations}

A CTA and baseline NIHSS has been included in the initial stroke investigation so that large vessel occlusion can be identified as early as possible and a thrombectomy indicated and performed sooner to reduce $\mathrm{mRS}$ and risk of complications. However, this may delay the administration of IVT and lead to further ischaemic damage, which may lower 
the ASPECT score and contraindicating thrombectomy. ESO recommendations state 'mechanical thrombectomy should not prevent the initiation of IVT where it is indicated, and IVT should not delay mechanical thrombectomy' [19]. This is a difficult to implement practically because currently thrombectomy is only used after IVT is administered, however thrombectomy is indicated with a large vessel occlusion only and this needs to be investigated initially before the clot is thrombolysed.

\section{Conclusion}

In summary, there is very little guidance in the UK for thrombectomy use in ischaemic stroke as evidenced by NICE guidance, which is vague, restrictive and based on older, smaller and less robust studies. ESO, on the other hand, uses stronger, more recent data to support its comprehensive guidance. The UK needs to update its guidelines in accordance to the new literature available and current European practice, both of which are incorporated into the proposed guideline. Furthermore, research needs to be carried out into the use of thrombectomy alone in large vessel strokes versus combination therapy (IVT + thrombectomy) as it can free up a great amount of time and financial resources. With the growing evidence to support thrombectomy, and the limitations of IVT therapy, it seems that we are headed towards a future lead by advances in interventional radiology; we can only hope that the UK based guidelines and practices can catch up with the European and international community on stroke management.

\section{References}

1. Sacco RL (2013) An updated definition of stroke for the $21^{\text {st }}$ Century. American Heart Association 44: 2064-2089.

2. Townsend N (2012) Coronary heart disease statistics. British Heart Foundation, London.

3. Stroke Association (2015) State of the Nation Stroke Statistics.

4. Saka O, McGuire A, Wolfe C (2009) Cost of stroke in the UK. Age and Ageing 38: 27-32.

5. Kumar V, Abbas AK, Aster JC (2012) Robbins basic pathology. Canada: Elsevier Saudners 2012: 814-816.

6. DiNuzzo M, Maraviglia B, Giove F (2011) Why the brain does not store glycogen Bioessays 33: 319-326

7. Longo DL (2011) Harrison's principles of internal medicine. McGraw-Hill 2011: 2560-2562

8. Lindsay KW, Bone I (2004) Neurology and Neurosurgery Illustrated. London: Churchill Livington 2004: 238-243.

9. Bamford JM (2000) The role of the clinical examination in the sub-classification of stroke. Cardiovascular Diseases 10: 2-4.

10. Raithatha A, Pratt G, Rash A (2013) Developments in the managemnt of acute ischaemic stroke. Continuing education in anaesthia. Critical Care and Pain 13: 80-86.

11. Merwick A, Werring D (2014) Posterior circulation ischaemic stroke-Clinical review. BMJ pp: 348.
12. National Institute of Health (2015) National Institute of Neurological Disorders and Stroke. Stroke Scale.

13. Runde D (2015) Modified Rankin Scale for neurologic disability. mRS-9Q MD calculator. Retrieved on 17-02-2015.

14. Intercollegiate Stroke Working Party (2012) National clinical guideline for stroke, (4thedn). London: Royal College of Physicians. p: 1-232.

15. NICE (2008) Stroke: Diagnosis and initial management of acute stroke and transient ischaemic attack (TIA). p: 1-29.

16. Grigoryan M, Qureshi Al (2010) Acute stroke management: Endovascular options for treatment. Semin Neurol 30: 469-476

17. Raychev R, Saver JL (2012) Mechanical thrombectomy devices for treatment of stroke. Neurol Clin Pract 2: 231-235.

18. Rohde S, Bösel J, Hacke W, Bendszus M (2012) Stent retriever technology: Concept, application and initial results. J Neuro Intervent Surg 4: 455-458.

19. ESO, ESMINT, ESNR, ESO-Karolinska Stroke Update (2014): A Consensus statement on mechanical thrombectomy. European Stroke Organization.

20. NICE (2013) Mechanical clot retrieval for treating acute ischaemic stroke. NICE interventional procedure guidance 548 .

21. Sekhar A (2015) Intra-arterial treatment in acute ischaemic stroke: What we learn from the MR CLEAN trial'. Journal of Royal College of Physicians of Edinburgh 45: 43-44.

22. Berkhemer OA (2015) A randomized trial of intraarterial treatment for acute ischemic stroke. N Engl J Med 372: 11-20.

23. Campbell BC (2015) Endovascular therapy for ischemic stroke with perfusion-imaging selection. N Engl J Med 372: 1009-1018.

24. Goyal M (2015) Randomized assessment of rapid endovascular treatment of ischemic stroke. N Engl J Med 372: 1019-1030.

25. Saver JL (2015) Stent-Retriever Thrombectomy after Intravenous t-PA vs. t-PA Alone in Stroke. N Engl J Med 372: 2285-2295.

26. Kumar G, Shahripour RB, Alexandrov AV (2014) Recanalization of acute basilar artery occlusion improves outcomes: A metaanalysis. J Neurointerv Surg 7(12): 868-874.

27. Cooray C, Fekete K, Mikulik R, Lees KR, Wahlgren N, et al. (2015) Threshold for NIH stroke scale in predicting vessel occlusion and functional outcome after stroke thrombolysis. Official Journal of the International Stroke Society. Int J Stroke 10: 822-829.

28. Heldner MR, Zubler C, Mattle HP, Schroth G, Weck A, et al. (2013) National institutes of health stroke scale score and vessel occlusion in 2152 patients with acute ischemic stroke. Stroke 44: 1153-1157

29. Rouchaud A, Mazighi M, Labreuche J (2011) Outcomes of mechanical endovascular therapy for acute ischemic stroke: a clinical registry study and systematic review. Stroke 42: 12891294.

30. Broderick JP, Palesch YY, Demchuk AM, Yeatts SD (2013) Endovascular therapy after intravenous t-PA versus t-PA alone for stroke. N Engl J Med 368: 893-903.

31. Dávalos A, Pereira VM, Chapot R (2012) Retrospective multicenter study of solitaire FR for revascularization in the treatment of acute ischemic stroke. Stroke 43: 2699-2705.

32. Rankin J (1957) Cerebral vascular accidents in patients over the age of 60. Scott Med J 2: 200-15. 
33. Ver Hage A (2011) The NIH stroke scale: a window into neurological status. Nurse.Com Nursing Spectrum 24: 44-49.

34. Saver JL (2015) Stent-retriever thrombectomy after intravenous t-PA vs. t-PA Alone in Stroke. N Engl J Med 372: 24

35. Sekhar A, Fletcher NA (2015) Guidelines of thrombectomy in large vessel occlusive stroke at the Walton Centre NHS Foundation Trust.
36. McDonald JS, Brinjikji W, Rabinstein AA, Cloft HJ, Lanzino G, et al. (2015) Conscious sedation versus general anaesthesia during mechanical thrombectomy for stroke: A propensity score analysis. J Neurointerv Surg 7: 789-794.

37. Behme D, Gondecki L, Fiethen S, Kowoll A, Mpotsaris A, et al. (2014) Complications of mechanical thrombectomy for acute ischemic stroke-a retrospective single-center study of 176 consecutive cases. Neuroradiology 56: 467-476. 